\title{
Using Waist Circumference Measurements to Enhance the Public's Understanding of Childhood Obesity: Accounting for Health Literacy
}

\author{
Madelyn P. Law ${ }^{1, *}$, John Hay ${ }^{1}$, Brent E. Faught ${ }^{1}$, John Cairney ${ }^{2}$ \\ ${ }^{1}$ Department of Health Sciences, Brock University, St. Catharines, Ontario, Canada, L2S3A1 \\ ${ }^{2}$ Department of Family Medicine, McMaster University, McMaster Innovation Park, 175 Longwood Road South, Suite 201A, Hamilton, \\ Ontario, Canada, L8P 0A1
}

Copyright (C) 2015 Horizon Research Publishing All rights reserved.

\begin{abstract}
This study examined the extent to which the measurement of waist circumference (WC) can categorize overweight/obesity status similarly to BMI in children. Height and weight were measured and BMI was calculated for participants' age 9 to 11 years old. Children were classified as either normal-weight, overweight, or obese. WC was measured twice and the average of these measures recorded. Receiver-operator characteristic curves were computed for each age and gender for both overweight and obese using BMI as the standard. For each analysis the area under the curve was used as an overall indicator of agreement between the two measures. WC achieved very strong sensitivity for both overweight and obesity for both genders, ranging from .90 to .93 . Specificity for obesity for both genders was very strong, ranging from .89 to .93 for girls and from .90 to .93 for boys. While specificity for obese remains high for both genders $(.89-.93)$ across all three years, the specificity for overweight fell to .68 to .84 for boys and to .66 to .74 for girls. Due to low levels of health literacy in the population, using $\mathrm{WC}$ may provide an easy to understand measure of obese or overweight status.
\end{abstract}

Keywords Health Literacy, Obesity, Body Mass Index, Waist Circumference

\section{Introduction}

In order for broad health promotion or disease prevention campaigns to be effective, it is essential that risk factors or symptoms are easily understood and recognizable to populations with low literacy levels. For example, reference to dizziness rather than vertigo, or chest pain rather than angina heighten individuals understanding and awareness of the need to seek immediate treatment for stroke or heart attack. Targeting specific health topics such as these through the use of plain language and easily understood measures helps to promote the identification of risk factors and the behavior required to reduce the risk of negative health outcomes. Researchers have outlined that chronic disease management requires a clear understanding and assessment of health information in order to enable change; namely focusing on the importance of health literacy [1-3].

We know that adults from Organization for Economic Co-operation and Development (OECD) member countries demonstrate wide variation in health status and literacy rates. Individuals classified as "most healthy" have higher average literacy scores than those who are classified as "least healthy" who have the lowest average literacy rates [4]. More specifically in Canada, health literacy rates are low, which mirrors the average literacy and numeracy skills [5]. When low rates of health literacy are juxtaposed with the ever-growing global health concern of childhood obesity, it is clear that health promoters need to be able to communicate with parents and children with language and measures they can understand. This understanding will in turn allow them to recognize the need to engage in solutions or seek assistance to deal with overweight/obesity. The following paper will suggest that an accepted, evidence-based method, to determine obesity and overweight status is waist circumference (WC), as it may serve that purpose for populations with low levels of health literacy. WC or waist size may be a means to provide information to children and parents in a commonly understood metric thus allowing them to easily and conclusively determine if their children are in fact overweight or obese.

The concept of health literacy has been defined in a number of different ways [6], which has also been paralleled with studies focused on varying methods and paradigms to understand the concept [7]. There is no universally accepted definition of health literacy used in research and practice. However, in a systematic review of available definitions, Sorenson et al.[7] proposed an all-inclusive definition that captures the essence of 17 definitions to understand health literacy: 
"Health literacy is linked to literacy and entails people's knowledge, motivation and competences to access, understand, appraise and apply health information in order to make judgments and take decisions in everyday life concerning healthcare, disease prevention and health promotion to maintain or improve quality of life during the life course" (p.3) [7].

Studies highlighted by the Institute of Medicine in the United States outline that $47 \%$ of adults in the United States lack the basic literacy skills necessary to understand and use health information [8]. Approximately 9 out of 10 American adults have difficulty acting on health information provided through health care organizations, community, and other health related channels [9]. This is mirrored, although to a lesser extent, in the Canadian population where $60 \%$ of Canadians lack the capacity to obtain, understand, and act on health information or to independently make appropriate decisions for their health [5]. Similar rates are found in the United Kingdom (46\%) and Australia (57\%) with citizens having lower levels of literacy which impact their ability to achieve optimal health $[10,11]$.

Health literacy is an increasingly important skill as medical advances become embedded within a complex health care system, and due to the exponential increase in access to health information. A failure by health promoters to recognize both the general literacy and health literacy of the target population will significantly impede success of achieving behavior change. Using simple and clear language with understandable and recognizable behavioral responses helps ensure messages will be understood and that behavior change can take place [12]. Using highly medicalized terminology and technical units or terms, while perfectly clear to the medical community, does not benefit or inform the lay public. There is evidence that a lack of clear information and understanding of disease prevention behaviours leads to individuals skipping medical tests, [9] increased emergency room visits, and difficulty managing chronic conditions [8].

Statistics Canada[13] reports that $21.8 \%$ of adolescents age 12-17 years old self report weights that are considered to be overweight or obese, which has been seen to be a rising trend in Canada. Surprisingly, campaigns to prevent and treat overweight and obese individuals, perhaps the single largest threat to population health [14], have not been able to promote to parents and children a simple and easily understood risk marker that would help to increase the public's identification of "being overweight or obese".

Predominant in the research literature, in clinical guidelines, and in communication of risk to the public, is the use of the Body Mass Index or BMI. This concept, developed to provide a simple and quick proxy for percentage of body fat, has achieved almost universal acceptance in spite of its well known flaws within the scientific community including, but not limited to, calculation errors, measurement errors, limited construct validity, poor specificity and sensitivity [15]. Its pervasiveness in the research literature is extraordinary given that it provides arbitrary units of measurement of area $\left(\mathrm{m} / \mathrm{kg}^{2}\right)$ as a proxy for volume and collapses all mass compartments - fat, muscle, bone, water into a single measure. Its widespread use in public health programs is equally puzzling as not only are the units meaningless to the layperson, but its computation requires two measurements and two mathematical steps which are not easily completed. Even where simple means of computing BMI are available (e.g., website, apps), these still require that individuals accurately take two measurements, and transpose the computed BMI value to a table to determine their category. These systems are also typically provided with riders that note the limitations of BMI as a measure of body fat, providing an easy escape for those who would prefer to deny their own, or their children's overweight status. In children specifically, BMI as a measure of body fat is problematic due to age, sex and pubertal maturation influences [16]. As a result, BMI appears difficult to understand, interpret, or be accepted by the general public. As both overweight/obesity and poor literacy are significantly higher among lower socioeconomic status, grouping this leaves those developing promotion programs for the highest risk groups in society bereft of an effective communication tool [17-19].

BMI is not without its redeeming qualities. When used as a proxy for adiposity in epidemiological studies, it has proven to be an effective measure in the determination of population risk [20]. Its correlation with body fat in large cohorts is sufficiently strong to make its usage reasonable in population studies [20], and it can be considered a reasonable guideline for use by clinicians in pediatric settings [21]. BMI however may not be intuitively meaningful to most individuals, particularly when used outside the context of clinical or research environments and in the general public with low health literacy levels. It should not be deemed as the only way in which to describe or recognize overweight or obesity.

The question then arises - is it possible to find a marker of overweight/obesity that meets four main tests: 1.easily recognized, understood, and accepted by the general population; 2.accessible and easy to apply by the public; 3.applicable and meaningful on an individual level for both clinicians and the general public; and 4 . accepted by the research and clinical communities as having a utility at least comparable to BMI for population studies. Meshing these considerations together to find a measurement that is scientifically accurate and addresses our understanding of health literacy is an essential requirement moving forward in practice to combat obesity with children.

The potential of using WC as a proxy measure of overweight/obesity demonstrates numerous opportunities for gaining entry in to the public consciousness about obesity. Waist size is an easily recognized concept, related to a common behavior - selecting clothes that fit, and straightforward to measure. Further it is widely and commonly used as an obvious sign of overweight. In 
common vernacular terms such as 'big belly' and 'spare tire' are all synonymous with overweight and are not conducive to rationalizing arguments of "big-boned" or "husky". Thus, a strong cognitive association with waist size and overweight/obesity already exists within the general public. As such, waist size would seem to meet test one and partially meet tests two and three above. WC has also become increasingly accepted and recognized by clinicians and the research community as a significant risk factor/marker for several chronic diseases including some cancers [22-24], diabetes [25], and cardiovascular disease in adult populations [26-27]. In fact, Janssen et al.[28] demonstrated that WC "is a better marker of obesity-related health risk than is BMI, and consequently a greater emphasis should be placed on WC in the obesity classification system" in an adult population (p. 381). However, unless WC can be demonstrated to be as useful as BMI in the child population cohort, it would not fulfill the demands of test four.

Therefore, this study will examine the extent to which the measurement of WC can predict overweight/obesity (OW/OB) similarly to BMI in children. To accomplish this, we used BMI as the criterion standard and employed Receiver-Operator Characteristic (ROC) curve analyses to determine the sensitivity and specificity of WC in determining overweight/obesity among a population. Should WC demonstrate very high specificity and sensitivity in the classification of $\mathrm{OW} / \mathrm{OB}$ when $\mathrm{BMI}$ is the criterion standard, this would provide evidence that WC cut-points could be used to replace BMI cut-points with little loss in precision.

\section{Materials and Methods}

\subsection{Data Collection}

The study population comprised all children in the fourth grade (ages 9 and 10) in the 2004/05 school year in the public school system in the Niagara region of Ontario, Canada participating in a larger longitudinal investigation, the Physical Health Activity Study Team (PHAST) study [29]. We received permission from $75(83 \%)$ of 92 possible schools to enroll students. Informed consent from the parents of $2278(96 \%)$ of 2378 children at these schools was obtained at baseline. We established testing and training protocols, assembled a cadre of trained assistants, developed procedures, and completed pilot testing in the fall of 2004 . Formal data collection began in the spring of 2005 when children were in the last semester of Grade Four and continued biannually for the following two years (Grades Five and Six). Ethics approval for the study was obtained from the local district school board and Brock University Research Ethics Board. This analysis reports results from children taken from the final semesters of Grades Four, Five, and Six. The sample consists of 1146 boys and 1113 girls in Grade Four ( $M$ age 9.9), 1147 boys and 1107 girls in Grade Five ( $M$ age 10.9), and 1072 boys and 1069 girls in Grade
Six ( $M$ age 11.9) for whom we had complete data.

\subsection{Procedures}

Height and weight were measured using portable stadiometer (SECA, Hamburg, Germany) and precise electronic weight scales (Tanita, Tokyo, Japan). WC was measured midway between the lowest rib and the superior border of the iliac crest [30]. The children's WC was measured three times over a light t-shirt and the average of these measures was recorded. This was done by trained research assistants and the average was taken. Body Mass Index (BMI) was calculated for each child and children were classified (by age in months and gender using percentiles provided by the Center for Disease Control) as either normal-weight, overweight (OW $-85^{\text {th }}$ - 94 th $)$, or obese (OB $-95^{\text {th }}$ and above). Using these cut-points 176 boys and 169 girls were OW and 156 boys and 140 girls were OB in Grade Four (age 9); 183 boys and 143 girls were OW and 150 boys and 134 girls were OB in Grade Five (age 10); and 198 boys and 158 girls were $\mathrm{OW}$ and 153 boys and 131 girls were $\mathrm{OB}$ in Grade Six (age 11). Analyses of variance revealed no significant gender differences in BMI but BMI did increase significantly by Grade: $18.8(S E=0.14)-19.2(S E=0.15)-$ $20.1(S E=0.15)$.

\subsection{Data Analysis}

Receiver Operating Characteristics (ROC) curves are used to determine the accuracy of a diagnostic test or screening tool based on its sensitivity and specificity. In order to determine the utility of WC as a measure of obesity ROC curves were computed for each grade and gender for both OW and OB status with that status determined using BMI as the standard. For each analysis the area under the curve (AUC) was used as an overall indicator of agreement between the two measures with $100 \%$ indicating complete agreement. Since WC is a continuous measurement it is necessary to determine cutpoints which classify a child as OW or OB. For each grade, gender, and OW/OB status, optimal sensitivity (rate of true positives) and specificity (rate of true negatives) WC cutoffs were determined by establishing the point where sensitivity reached at least $90 \%$. At this point at least $90 \%$ of all children identified as $\mathrm{OW}$ or OB using BMI cutoffs would have been similarly diagnosed as OW or OB using the assigned WC cutoff.

\section{Results}

The results of the analyses for the AUC are presented in Table 1. AUC can be seen as an indication of the degree of agreement between the two measures and it is evident that there is very good agreement for $\mathrm{OW}$ and very strong agreement for OB over all three years and for both genders. The outstanding agreement between the two body composition assessments measures for OB (.97-.98) 
provides strong evidence that the two measures are virtually interchangeable. There is greater variability in AUC estimates for OW (.79-.98), suggesting that there is greater risk for disagreement for this clinical marker. However, even the low end estimate for AUC (.79) is still very good.

Table 1. ROC - Area under the Curve Percentage (CI) - males and females Grade 4-6

\begin{tabular}{|c|c|c|c|}
\hline & OW & OB & OW/OB \\
\hline Grade 4 & & & \\
\hline Boys & $.79(.014)$ & $.98(.004)$ & $.87(.008)$ \\
\hline Girls & $.78(.014)$ & $.97(.008)$ & $.93(.008)$ \\
\hline Grade 5 & & & \\
\hline Boys & $.81(0.13)$ & $.99(.003$ & $.89(.007)$ \\
\hline Girls & $.83(.012)$ & $.98(.004)$ & $.97(.004)$ \\
\hline Grade 6 & & & \\
\hline Boys & $.77(.019)$ & $.98(.004)$ & $.87(.008)$ \\
\hline Girls & $.98(.004)$ & $.98(.004)$ & $.95(.007)$ \\
\hline
\end{tabular}

The results for sensitivity and specificity are provided in Table 2. Sensitivity refers to the probability that the new assessment tool provides a positive result given that BMI has determined the individual to be OW or OB. Specificity refers to the probability of providing a negative result given that $\mathrm{BMI}$ has deemed the individual not OW or OB. As capturing all persons at risk is of significant importance and the harm of a false negative fairly low in this situation, in this analysis the decision was made to set a sensitivity target of at least .90 when establishing a WC cutoff.

Table 2. ROC -Sensitivity and Specificity - males and females, Grades 4-6

\begin{tabular}{|c|c|c|c|c|}
\hline & Sensitivity & Specificity & $\begin{array}{c}\text { Cutoff } \\
\text { cm/inches }\end{array}$ & $\begin{array}{c}\text { Approximate } \\
\text { Clothing Size }\end{array}$ \\
\hline Grade 4 & & & & \\
\hline FOW & .91 & .67 & $63.5 / 25$ & $12 / 14$ \\
\hline FOB & .93 & .89 & $72.9 / 28.7$ & $14 / 16$ \\
\hline MOW & .91 & .84 & $64 / 25.2$ & 10 \\
\hline MOB & .92 & .92 & $73 / 28.7$ & 18 \\
\hline Grade 5 & & & & \\
\hline FOW & .90 & .74 & $71.1 / 28.0$ & $14 / 16$ \\
\hline FOB & .91 & .93 & $79.5 / 31.3$ & 16 \\
\hline MOW & .92 & .73 & $69 / 27.2$ & 14 \\
\hline MOB & .92 & .90 & $77.5 / 30.5$ & 22 \\
\hline Grade 6 & & & & \\
\hline FOW & .91 & .66 & $71.4 / 28.1$ & $14 / 16$ \\
\hline FOB & .90 & .93 & $81.6 / 32.1$ & 16 \\
\hline MOW & .92 & .68 & $71.0 / 28.0$ & 18 \\
\hline MOB & .91 & .93 & $81.4 / 32.0$ & 22 \\
\hline
\end{tabular}

It is clear that $\mathrm{WC}$ was able to achieve a very strong sensitivity for both OW and OB for both genders, ranging from $.90-.93$. Specificity for OB for both genders was also very strong, ranging from .89 to .93 for girls and from .90 to .93 for boys. This means that at least 90 of 100 children classed as obese or overweight using BMI would be correctly identified using the given WC cutoffs for age and gender, while the remaining 10 children would be classified as normal weight who were not identified as such using BMI. Using high sensitivity cutoffs in an attempt to capture all those with a condition typically results in a cost to the precision of specificity and this is the case to some extent here. While specificity for OB remains high for both genders (.89 - .93) across all three years, the specificity for OW ranges from .68 to .84 for boys and from .66 to .74 for girls. This suggests that a larger number of children would be classified as OW using WC opposed to BMI as a criterion.

Table 2 also provides an approximation of clothing (pant) sizes in the final column to help equate the WC measurement to size.

\section{Discussion}

The results presented here suggest that there is excellent sensitivity and specificity for $\mathrm{OB}$, and excellent sensitivity with moderate specificity for OW when using WC to predict these based on BMI criteria. In this instance and from a public health perspective, the serious consequences of poor sensitivity (a false negative or missed diagnosis) outweigh the potential harm resulting to those mis-diagnosed due to weaker specificity (false positive). These findings appear to meet the demands of our fourth assessment criterion in that $\mathrm{WC}$ is at least as efficient in identifying OW/OB as BMI. At some juncture, the WC cutoffs in $\mathrm{cm} / \mathrm{in}$, which could be easily measured by both professionals and laypersons, could be replaced by approximately equivalent waist/clothes sizes in public health campaigns to allow a deeper penetration into the mindset of the public. For example, a 10-year-old boy wearing jeans with a 34 " waist would be indicated as obese. Although jean sizes may vary by brand type, this variance is not so great to invalidate this potential approach.

This is a trial using a single large data set to explore the potential of using WC, potentially expressed as jean size as an alternative for parents in determining if their children are at risk of OW/ OB. The existing measure, BMI, is complex to measure and devoid of meaningful units and demands a high standard of health literacy. Knowing what jean size is comfortable to wear is simple and universal. There is a great deal of literature expressing strong correlations between $\mathrm{BMI}$ and $\mathrm{WC}$ and there is good reason to believe that similarly strong ROC analysis results will be evident in other populations and age groups. This is the only large scale attempt to determine quasi-diagnostic cut offs for OW and $\mathrm{OB}$ using $\mathrm{WC}$ with $\mathrm{BMI}$ as the reference. Existing national datasets could be employed to develop WC cutoffs by age for OW/OB that would be widely generalizable, something that the dataset employed here is unable to achieve.

\subsection{Limitations and Future Directions}


The main limitation of this study is that the analysis was limited to children ages nine to eleven from a single geographic region's local schools which limits the generalizability to other areas. As well, the extent to which these results could be replicated with other age groups is not known. However, it is important to note the potential of using WC markers for the purposes of enhancing awareness and understanding of $\mathrm{OW}$ and $\mathrm{OB}$ as this tool has many attractive health literacy properties. This limitation provides a challenge for future research to examine whether these findings hold true in other age groups and populations hold true. The end result might be a simple chart relating waist size and age to provide a risk of OW/OB that would be meaningful to the general population and providing a simple means for clinicians to counsel parents regarding healthy weights. It is also important to note that $\mathrm{WC}$ is only one of many ways to evaluate OW and OB. Others methods such as waist-hip ratio, subcutaneous fat depth, and BMI can also be used, but all have associated advantages and limitations. Using WC does only provide an estimation or general idea of $\mathrm{OW}$ and $\mathrm{OB}$, and does not equal a medical diagnosis. Obesity may be seen as intra-dominal or subcutaneous obese, healthy obese and unhealthy obese all of which would require a medical assessment.

\subsection{Implications}

Both public health and medical professionals alike must address major health concerns through evidence-based methods. Having a measure that is both scientifically valid and addresses concerns of health behavior change with a population with low health literacy is of paramount importance. The ability of individuals to conduct an assessment of obesity in "the comfort of their own home" that is scientifically proven and requires minimal interpretation is a major stride forward to combating this epidemic with children. While BMI can also be calculated at home it still requires two measures, access to the Internet and/or skill in using calculators, and making sense of a number with no recognizable units. Having a measurement technique that is easily understood and accessible to the parents will provide a starting point for individuals to become aware of their current physical state, which in turn, may help to motivate them to act on this information and make positive health behavior changes. Incorporating this measurement in to current health promotion plans and programs, which contend with obesity in children, may provide for a greater level of engagement from the public given their ability to understand $\mathrm{OW}$ and $\mathrm{OB}$ benchmarks.

The use of WC also links to the challenges of family practitioners. BMI is a difficult clinical guideline for physicians to take in their practice due to appointment time constraints, but with evidence that $\mathrm{WC}$ is a valid measure of obesity; it is possible that this faster less complicated technique could be implemented into the clinical setting. Doctors, physician assistants, and nurse practitioners could conduct this measurement and discuss with children and parents their results based on scientific guidelines to highlight their risk factors. Using a measure such as WC becomes much less intimidating than the BMI, which requires greater levels of health literacy from the patient's perspective.

\section{REFERENCES}

[1] Lloyd, L., Ammary, N., Epstein, L., Johnson, R., Rhee, K. A transdisciplinary approach to improve health literacy and reduce disparities, Health Promotion Practice, Vol.7, No.3, 331-335, 2006

[2] Osborne, H. Health Literacy: From A to Z (2nd ed.), Jones \& Bartlett Learning, Burlington, Massachusetts, 2011.

[3] Mitic, W., Rootman, I. Inter-sectoral approach to improving health literacy for Canadians, Online available from http://www.phabc.org/modules.php?name=Contentpub\&pa= showpage\&pid=182.

[4] Minister of Industry, Canada and Organisation for Economic Cooperation and Development (OECD). Learning a Living: First Results of the Adult Literacy and Life Skills Survey, Online available fromhttp://www.oecd.org/education/educationeconomyands ociety/34867438.pdf.

[5] Canadian Council of Learning. Health Literacy in Canada, Online available from http://www.ccl-cca.ca/pdfs/HealthLiteracy/HealthLiteracyRe portFeb2008E.pdf.

[6] Berkman, N.D., Davis, T.C., McCormack, L. Health literacy: What is it?, Journal of Health Communications, Vol.15, No.2, 9-19, 2010.

[7] Sorenson, K., Van den Broucke S., Fullarn, J., Doyle, G., Pelikan, J., Slonska, Z., Brand, H., Consortium Health Literacy Project European. Health literacy and public health: A systematic review and integration of definitions and models, BMC Public Health, Vol.12, No.80, 1471-2458, 2012.

[8] Nielsen-Bohlman, L., Panzer, A.M., Kindig, D.A. Health Literacy: A Prescription to End Confusion: Committee on Health Literacy, The National Academies Press, Washington, DC, 2004.

[9] Kutner, M., Greenberg, E., Jin, Y., Paulsen, C. The Health Literacy of America's Adults: Results from the 2003 National Assessment of Adult Literacy (NCES 2006-483), 2006.

[10] Williams, J., Clemens, S., Oleinikova, K., Tarvin, K. The skills for life survey. A national needs and impact survey of literacy, numeracy and ICT skills, Department for Education and Skills, London, 2003.

[11] Australian Bureau of Statistics. Health Literacy, Australia. Online available from http://www.abs.gov.au/ausstats/abs@.nsf/lookup/4102.0mai $\mathrm{n}+$ features20june +2009 .

[12] Berkman, N., Sheridan, S., Donahue, K., Halpern, D., Viera, A., Crotty, K., Holland, A., Brasure M., Lohr, K., Harden, E., Taut, E., Wallace, I., Viswanathan, M. Health literacy interventions and outcomes: an updated systematic review, 
Evidence report/technology assessment no. 199. (Prepared by RTI International-University of North Carolina Evidence-based Practice Centre under contract No. 290-2007-10056-I.), Agency for Healthcare Research and Quality, Rockville, MD, 2011.

[13] Statistics Canada. Body mass index, overweight or obese, self-reported, youth, by sex, Online available from http://www.statcan.gc.ca/tables-tableaux/sum-som/101/cst01/ health 83 b-eng.htm.

[14] James, P.T., Leach, R., Kalamara, E., Shayeghi, M. The worldwide obesity epidemic, Obes Res, Vol.9, No.s11, 228S-233S, 2001.

[15] Rothman, K.J. BMI-related errors in the measurement of obesity, International Journal of Obesity, S56-S59, 2008.

[16] Neovius, M., Linné, Y., Rossner S. BMI, waist-circumference and waist-hip-ratio as diagnostic tests for fatness in adolescents, International Journal of Obesity, Vol.

[17] Ad Hoc Committee on Health Literacy for the Council on Scientific Affairs. Health literacy: report of the Council on Scientific Affairs, JAMA, Vol.281, 552-557, 1999.

[18] Kim, S.P., Knight, S.J., Tomori, C., Colella, K.M., Schoor, R.A., Shih, L., Bennett, C.L. Health literacy and shared decision making for prostate cancer patients with low socioeconomic status, Cancer Investigation, Vol.19, No.7, 684-691, 2001.

[19] Davis, T.C., Dolan, N.C., Ferreira, M.R., Tomori C, Green KW, Sipler AM, Bennett CL. The Role of Inadequate Health Literacy Skills in Colorectal Cancer Screening, Cancer Investigation, Vol.19, No.2, 193-200, 2001.

[20] Must, A., Anderson, S.E. Body mass index in children and adolescents: considerations for population-based applications, International Journal of Obesity, Vol.30, 590-594, 2006.

[21] Reilly, J.J. Diagnostic accuracy of the BMI for age in pediatrics, International Journal of Obesity, Vol.30, 595-597, 2006.

[22] Janisewski, P.M., Oeffinger, K.C., Church, T.S., Dunn, A.L., Eshelman, D.A., Victor, R.G., Brooks, S., Turoff, A.J., Sinclair, E., Murray, J.C., Bashore, L., Ross, R. Abdominal obesity, liver fat, and muscle composition in survivors of childhood acute lymphoblastic leukemia, Journal of Clinical Endocrinology \& Metabolism, Vol. 92, No.10, 3816-3821, 2007.

[23] Huang, Z., Willett, W.C., Colditz, G.A. Waist circumference, waist: hip ratio, and risk of breast cancer in the Nurses' Health Study, American Journal of Epidemiology, Vol.150, 13161324, 1999.

[24] Moore, L.L., Bradlee, M.L., Singer, M.R., Splansky, G.L., Proctor, M.H., Ellison, R.C., Kreger, B.E. BMI and waist circumference as predictors of lifetime colon cancer risk in Framingham Study adults, International Journal of Obesity, Vol.28, 559-567, 2004.

[25] Vasquez, G., Duval, S., Jacobs, Jr. D.R. \& Silventoinen, K. Comparison of body mass index, waist circumference, and waist/hip ratio in predicting incident diabetes: A meta-analysis, Epidemiologic Reviews, Vol.28, 2007.

[26] Rexrode, K.M., Buring, J.E., Manson, J.E. Abdominal and total adiposity and risk of coronary heart disease in men, Journal of the International Association for the Study of Obesity, Vol.25, No.7, 1047-1056, 2001.

[27] Han, T.S., van Leer, E.M., Seidell, J.C., Lean, M.E.J. Waist circumference action levels in the identification of cardiovascular risk factors: prevalence study in a random sample, BMJ, Vol.311, 1401, 1995.

[28] Janssen, I., Katzmarzyk, P.T., Ross, R. Waist circumference and not body mass index explains obesity-related health risk, American Journal of Clinical Nutrition, Vol.79, 379-384, 2004.

[29] Cairney, J., Hay, J., Veldhuizen, S., Missiuna, C., Mahlberg, N., Faught, B.E. Trajectories of relative weight and waist circumference among children with and without developmental coordination disorder, CMAJ, Vol.182, No.11, 1167-1172, 2010.

[30] Gillium, R.F. Distribution of waist-to-hip ratio, other indices of body fat distribution and obesity and associations with HDL cholesterol in children and young adults aged 4-19 years: the Third National Health and Nutrition Examination Survey, Int J Obes Relat Metab Disord, Vol.23, 556-63, 1999. 